\title{
Interpretation of ATLAS and CMS Higgs measurements in STXS and EFT
}

\author{
Nikita Belyaev ${ }^{a, *}$, on behalf of the ATLAS and CMS Collaborations \\ ${ }^{a}$ National Research Nuclear University MEPhI (Moscow Engineering Physics Institute), \\ Kashirskoe highway 31, Moscow, 115409, Russia \\ E-mail: Nikita.Belyaev@cern.ch, NLBelyayev@mephi.ru
}

Since the Higgs boson discovery in 2012, the ATLAS and CMS collaborations are performing intensive studies of its properties. Those studies include, among other things, measurements of cross sections, branching ratios, coupling constants and mass. In this paper, the current status of effective field theory interpretations of the simplified template cross section measurements is presented for different production modes and decay channels of the Higgs boson. Limits on the Higgs boson effective field theory couplings were set by using $13 \mathrm{TeV}$ proton-proton collisions data recorded by the ATLAS and CMS detectors at the LHC.

The Eighth Annual Conference on Large Hadron Collider Physics-LHCP2020

25-30 May, 2020

online

\footnotetext{
*Speaker
} 


\section{Introduction}

Higgs boson physics is one of the most important fields of study nowadays. Both the ATLAS [1] and CMS [2] collaborations performed many different measurements of the Higgs boson properties. The Simplified Template Cross Section (STXS) measurements [3], which are the most common types of results in ATLAS and CMS, are often used to probe the Higgs boson couplings. The STXS are physical production cross sections (in contrast to e.g. signal strengths). They are defined in mutually exclusive regions of phase space ("bins"). The STXS concept was developed in order to simultaneously maximize the experimental sensitivity and minimize the theoretical uncertainty of the measurements. The binning provides isolation of possible Beyond the Standard Model (BSM) effects. The STXS measurements are also suitable for global combinations. However, the STXS are not fully fiducial and do not take into account Higgs decay information.

The results of the STXS measurements can be interpreted in several ways. One of the most promising tools in this field is the Effective Field Theory (EFT) approach, in which the SM Lagrangian is supplemented by new operators with canonical dimension $D$ larger than 4 [4]:

$$
\mathcal{L}_{E F T}=\mathcal{L}_{S M}+\sum_{i} \frac{c_{i}^{(5)}}{\Lambda} O_{i}^{(5)}+\sum_{i} \frac{c_{i}^{(6)}}{\Lambda^{2}} O_{i}^{(6)}+\sum_{i} \frac{c_{i}^{(7)}}{\Lambda^{3}} O_{i}^{(7)}+\sum_{i} \frac{c_{i}^{(8)}}{\Lambda^{4}} O_{i}^{(8)}+\cdots,
$$

where $c_{i}$ are so-called Wilson coefficients and $O_{i}^{(D)}$ are the operators of dimension $D$. This framework can be used to interpret the STXS results (but also applicable for the interpretation of differential cross sections and kinematic measurements). The Higgs boson production cross section in STXS region $p$ thus can be expressed as:

$$
\sigma_{p}=\sigma_{p, S M}+\sigma_{p, \text { interference }}+\sigma_{p, B S M} .
$$

Its ratio to the $\mathrm{SM}$, in turn, can be then parameterized as follows [5]:

$$
\frac{\sigma_{p}}{\sigma_{p, S M}}=1+\sum_{i} A_{i}^{\sigma_{p}} c_{i}+\sum_{i j} B_{i j}^{\sigma_{p}} c_{i} c_{j}
$$

where $A_{i}^{\sigma_{p}}$ and $B_{i j}^{\sigma_{p}}$ are coefficients independent of the $c_{i}$ and determined from simulation.

EFT interpretations of STXS measurements presented in this paper were done using $139 \mathrm{fb}^{-1}$ of LHC proton-proton collision data at $\sqrt{s}=13 \mathrm{TeV}$ collected by the ATLAS and CMS experiments.

\section{Higgs boson EFT measurements with the ATLAS detector}

The ATLAS collaboration has performed studies of the Higgs boson properties in the fourlepton decay channel [6]. The Higgs boson candidate events were categorised into several topologies, providing sensitivity to different production modes in various regions of phase space. Additional multivariate discriminants were used to further improve the sensitivity in reconstructed event categories with a sufficiently large number of events. The cross section times branching ratio for the $H \rightarrow Z Z^{*}$ decay measured in dedicated production bins are in good agreement with the SM predictions. Based on the product of cross section, branching ratio and acceptance measured in Reduced Stage-1.1 production bins of STXS, constraints were placed on possible CP-even and CP-odd BSM interactions of the Higgs boson with vector bosons, gluons and top quarks within the SMEFT framework [4]. The results of these measurements are shown in Figure 1. 

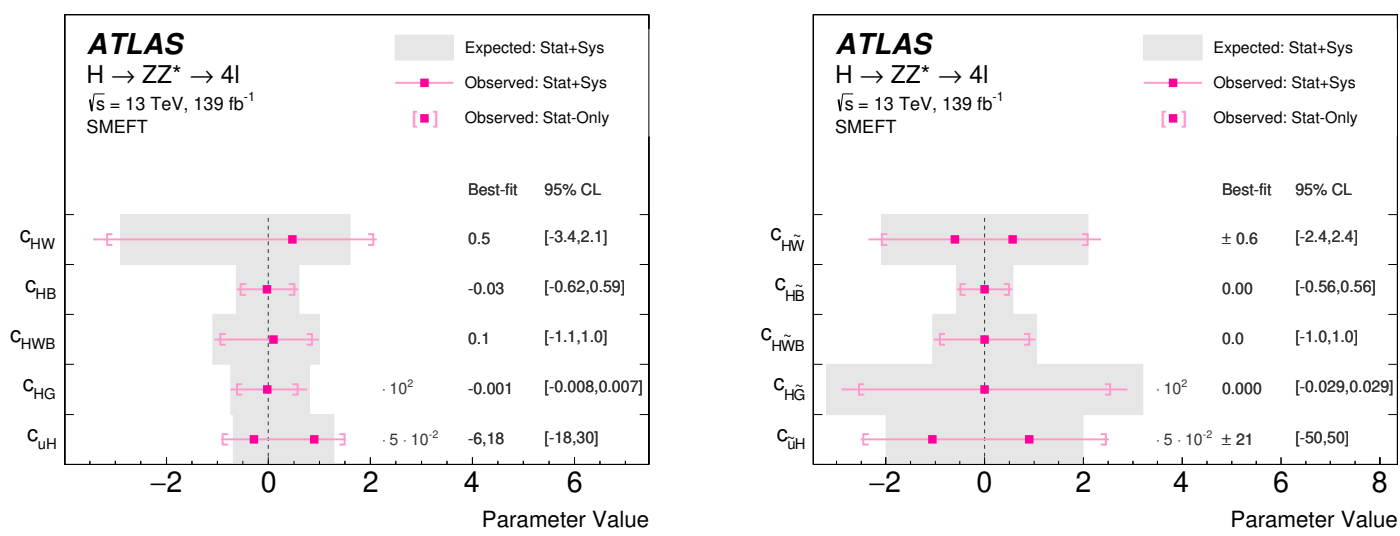

Figure 1: The observed and expected values of SMEFT Wilson coefficients from (left, [6]) CP-even and (right, [6]) CP-odd operators obtained for an integrated luminosity of $139 \mathrm{fb}^{-1}$ at $\sqrt{s}=13 \mathrm{TeV}$. Only one Wilson coefficient is fitted at a time while all others are set to zero. The values for the $C_{H G}$ and $C_{H \tilde{G}}$ coefficients are scaled by a factor of 100 , and for the $C_{u H}$ and $C_{\tilde{u} H}$ coefficients by a factor of 0.05 . The horizontal bands represent the expected measurement uncertainty.

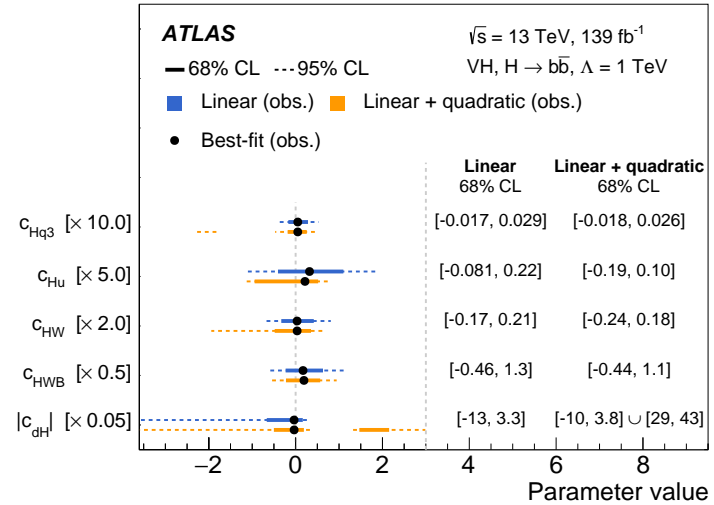

Figure 2: Summary of the observed best-fit values and one-dimensional confidence intervals for the Wilson coefficients of the Warsaw-basis operators to which this analysis has the greatest sensitivity along with the $c_{d H}$ coefficient which directly affects the $H \rightarrow b \bar{b}$ decay width. Limits are shown for the case where only linear (blue) or linear and quadratic (orange) terms are considered and confidence intervals are shown at both $68 \%$ CL (solid lines) and 95\% CL (dashed lines) [7].

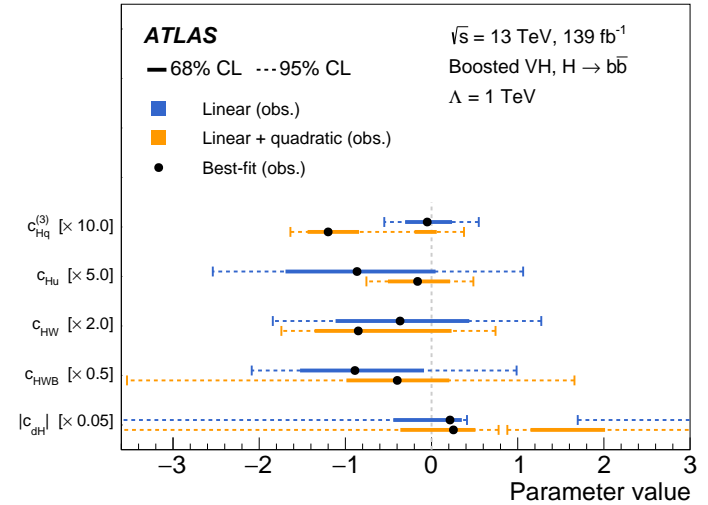

Figure 3: Summary of the observed individual confidence interval at 68\% (solid lines) and 95\% (dashed lines) CL for the $c_{H q}^{(3)}, c_{H u}, c_{H W}, c_{H W B}$ and $|c d H|$ Wilson coefficients from a fit of the STXS, using a linear-only parameterisation (in blue) and including quadratic terms (in orange) [8].

Properties of the Higgs boson decaying into a $b \bar{b}$ pair and produced in association with a $W$ or $Z$ boson were also studied [7]. Cross sections of associated production of a Higgs boson decaying into bottom quark pairs and an electroweak gauge boson, $W$ or $Z$, decaying into leptons were measured as a function of the gauge boson transverse momentum in kinematic fiducial volumes in the STXS 
framework. Limits were also set on the coefficients of effective Lagrangian operators which affect the $V H$ production and $H \rightarrow b \bar{b}$ decay. Limits were considered for both the variation of a single coefficient and also the simultaneous variation of a set of linear combinations of coefficients. The allowed range of the individual or linear combinations of the coefficients, to which the analysis has the greatest sensitivity, is limited to a few percent. The results are shown in Figure 2.

The $H b b$ analysis was also extended up to the region of high transverse momentum of the associated vector boson. These are the most precise measurements currently available in the high transverse momentum $\left(p_{\mathrm{T}}\right)$ regime for this process. The Higgs boson is reconstructed from a single large- $R$ jet to enhance the sensitivity in the high- $p_{\mathrm{T}}$ regime. Cross sections of associated production of a Higgs boson decaying into $b$ quark pairs with a $W$ or $Z$ gauge boson, decaying into leptons, were also measured in the STXS framework in two $p_{\mathrm{T}}^{V}$ regions: $250 \mathrm{GeV}<p_{\mathrm{T}}^{V}<400 \mathrm{GeV}$ and $p_{\mathrm{T}}^{V} \geq 400 \mathrm{GeV}$. All results were interpreted in terms of constraints on anomalous couplings in the framework of a Standard Model effective field theory (SMEFT). The results are shown in Figure 3.

\section{Higgs boson EFT measurements with the CMS experiment}

The CMS collaboration performed combined measurements of the Higgs boson production and decay rates; coupling modifiers to the SM particles; and EFT interpretations in terms of the Higgs boson self coupling and parameters. Analyses targeting the gluon fusion $(\mathrm{ggF})$, vector boson fusion (VBF), $W-$, $Z$ - and $t t$-associated production modes were included in the combination. These analyses target $H$ decays to $\gamma \gamma, Z Z, W W, \tau \tau, b b$, and $\mu \mu$ pairs [9]. An EFT interpretation was done, in which constraints on the parameters of the Higgs Effective Lagrangian (HEL) model were determined and are shown in Figure 4, the regions outside the bands are excluded. For many of the parameters these results represent the strongest constraints to date.

The double minimum in the likelihood scan presented in Figure 4 is a result of a degeneracy in the $H \rightarrow b \bar{b}$ scaling function. For example, in case of $c_{d}$ such a degeneracy corresponds to the SM prediction at $c_{d}=0$ and $c_{d} \approx-1.3$. Including EFT variations in bbH production would alleviate this degener-

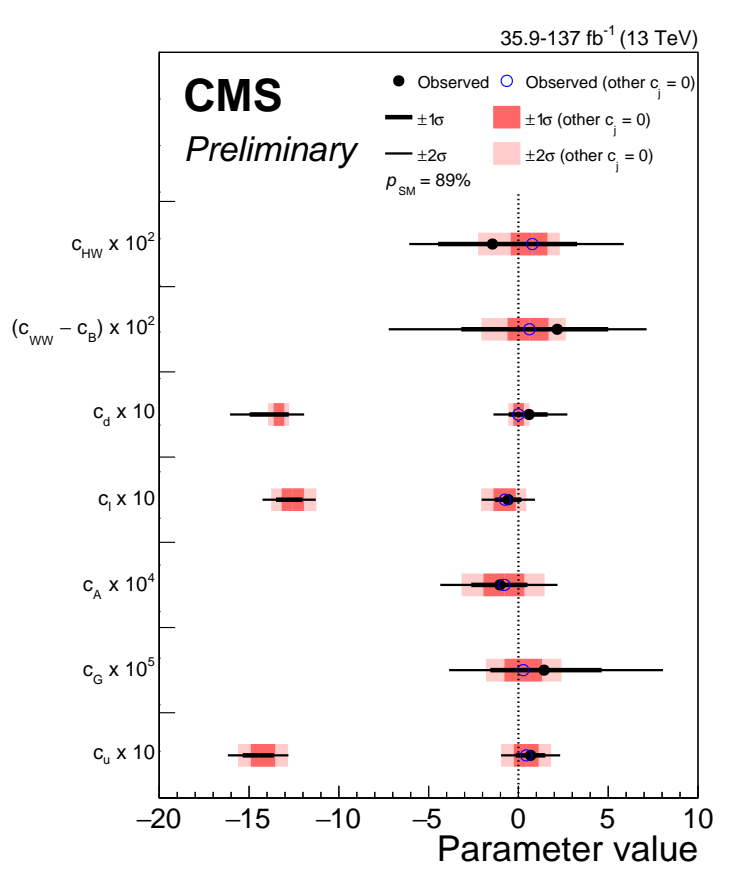

Figure 4: Summary plot for the HEL parameter scans. The best fit values when profiling (fixing) the other parameters are shown by the solid black (hollow blue) points. The $\pm 1 \sigma$ and $\pm 2 \sigma$ confidence intervals are represented by the thick and thin black lines respectively for the profiled scenario [9]. in the bbH parametrization. However, as described above, bbH production is fixed to the Standard Model prediction since there is no dedicated analysis category targeting this production mode. 
Another important study is related to anomalous $H V V$ interactions, including $\mathrm{CP}$ violation, using its associated production with two hadronic jets in $\mathrm{VBF}$, in the $V H$ process, and in $\mathrm{ggF}$, and subsequently decaying to a pair of $\tau$ leptons [10]. Constraints on the CP-violating parameter $f_{a 3}$ and on the CP-conserving parameters $f_{a 2}, f_{\Lambda 1}$, and $f_{\Lambda 1}^{Z \gamma}$ were set using matrix element techniques. The $68 \% \mathrm{CL}$ constraints are generally tighter than those from previous measurements using either production or decay information. Further constraints are obtained in the combination of the $H \rightarrow \tau \tau$ and $H \rightarrow 4 l$ decay [11] channels and are summarized in Figure 5.

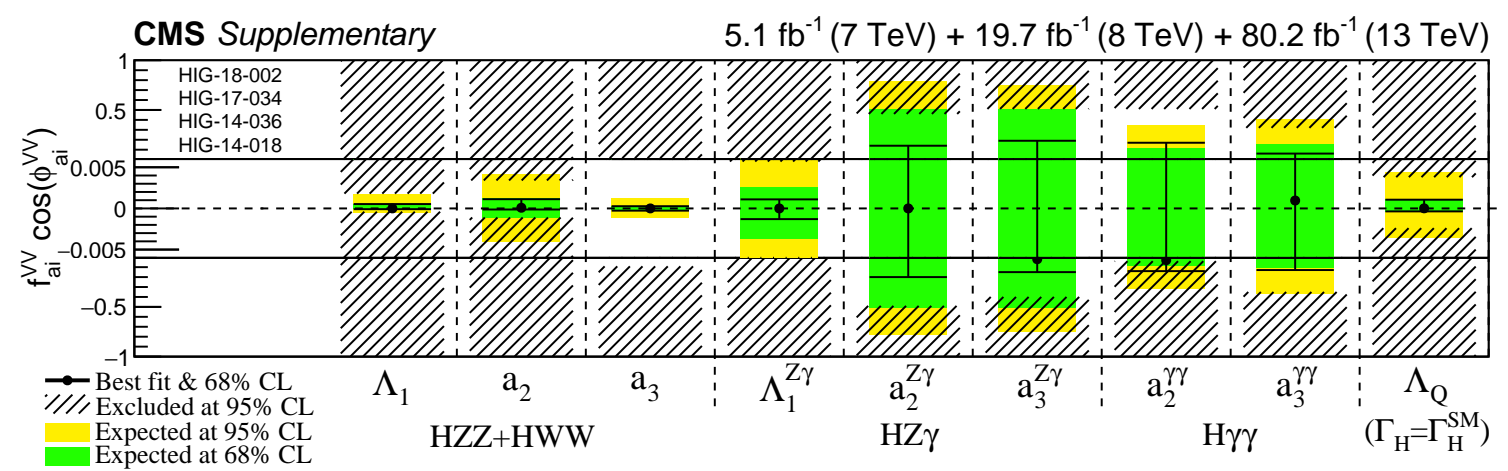

Figure 5: Summary of confidence level intervals of anomalous coupling parameters in $H V V$ interactions under the assumption that all coupling ratios are real $\left(\phi_{a i}^{V V}=0\right.$ or $\left.\pi\right)$ [10]. The $H Z Z+H W W$ coupling limits assume that $a_{i}^{Z Z}=a_{i}^{W W}$. The expected $68 \%$ and $95 \% \mathrm{CL}$ regions are shown as green and yellow bands. The observed intervals for $68 \% \mathrm{CL}$ are shown as points with error bars, and the hatched areas indicate the excluded regions at $95 \% \mathrm{CL}$. The limits on $f_{a 2,3}^{Z \gamma, \gamma \gamma}$ are from [12], and the limits on $f_{\Lambda Q}$ are from [13].

This combination places the most stringent constraints on anomalous $H$ boson couplings: $f_{a 3} \cos \phi_{a 3}=(0.00 \pm 0.27) \times 10^{-3}, f_{a 2} \cos \phi_{a 2}=\left(0.08_{-0.21}^{+1.04}\right) \times 10^{-3}, f_{\Lambda 1} \cos \phi_{\Lambda 1}=\left(0.00_{-0.09}^{+0.53}\right) \times$ $10^{-3}$, and $f_{\Lambda 1}^{Z \gamma} \cos \phi_{\Lambda 1}^{Z \gamma}=\left(0.0_{-1.3}^{+1.1}\right) \times 10^{-3}$. A simultaneous measurement of the $f_{a 3}$ and $f_{a 3}^{g g H}$ parameters was also performed, where the latter parameter is sensitive to $\mathrm{CP}$ violation effects in the gluon fusion process. However, the current data set does not allow for precise constraints on $\mathrm{CP}$ properties in the ggF process.

\section{Summary}

A number of recent analyses performed by the ATLAS and CMS collaborations has been presented. In those analyses, the simplified template cross section measurements were interpreted in terms of effective field theory. New constraints on the anomalous couplings of SMEFT and HEL Lagrangians have been set. The results are found to be consistent with expectations for the Standard Model Higgs boson.

\section{Acknowledgments}

The work was supported by the Ministry of Science and Higher Education of the Russian Federation, Project "Fundamental properties of elementary particles and cosmology" No 07232020-0041. 


\section{References}

[1] ATLAS Collaboration, The ATLAS Experiment at the CERN Large Hadron Collider, JINST 3 (2008) S08003 [10.1088/1748-0221/3/08/S08003].

[2] CMS Collaboration, The CMS Experiment at the CERN LHC, JINST 3 (2008) S08004 [10.1088/1748-0221/3/08/S08004].

[3] LHC Higgs Cross Section Working Group, Simplified Template Cross Sections - Stage 1.1, LHCHXSWG-2019-003 (2019) [arxiv:1906.02754].

[4] A. Falkowski, Higgs Basis: Proposal for an EFT basis choice for LHC HXSWG, LHCHXSWGINT-2015-001 (2015) [cds.cern.ch/record/2001958]

[5] ATLAS Collaboration, Methodology for EFT interpretation of Higgs boson Simplified Template Cross-section results in ATLAS, ATL-PHYS-PUB-2019-042 (2019) [cds.cern.ch/record/2694284].

[6] ATLAS Collaboration, Higgs boson production cross-section measurements and their EFT interpretation in the $4 \mathrm{l}$ decay channel at $\sqrt{s}=13 \mathrm{TeV}$ with the ATLAS detector, CERN-EP2020-034 (2020) [arxiv:2004.03447].

[7] ATLAS Collaboration, Measurement of $V H, H \rightarrow b \bar{b}$ production as a function of the vectorboson transverse momentum in $13 \mathrm{TeV}$ pp collisions with the ATLAS detector, JHEP 05 (2019) 141 [10.1007/JHEP05(2019)141].

[8] ATLAS Collaboration, Measurement of the associated production of a Higgs boson decaying into b-quarks with a vector boson at high transverse momentum in pp collisions at $\sqrt{s}=13$ TeV with the ATLAS detector, CERN-EP-2020-093 (2020) [arxiv:2008.02508].

[9] CMS Collaboration, Combined Higgs boson production and decay measurements with up to $137 \mathrm{fb}^{-1}$ of proton-proton collision data at $\sqrt{\mathrm{s}}=13 \mathrm{TeV}$, CMS-PAS-HIG-19-005 (2020) [cds.cern.ch/record/2706103].

[10] CMS Collaboration, Constraints on anomalous HVV couplings from the production of Higgs bosons decaying to $\tau$ lepton pairs, Phys. Rev. D 100 (2019) 112002 [CMS-HIG-17-034]

[11] CMS Collaboration, Measurements of the Higgs boson width and anomalous HVV couplings from on-shell and off-shell production in the four-lepton final state, Phys. Rev. D 99 (2019) 112003 [10.1103/PhysRevD.99.112003]

[12] CMS Collaboration, Constraints on the spin-parity and anomalous HVV couplings of the Higgs boson in proton collisions at 7 and 8 TeV, Phys. Rev. D 92 (2015) 012004 [10.1103/PhysRevD.92.012004].

[13] CMS Collaboration, Limits on the Higgs boson lifetime and width from its decay to four charged leptons, Phys. Rev. D 92 (2015) 072010 [10.1103/PhysRevD.92.072010]. 